\title{
Spectral domain optical coherence tomography: a better OCT imaging strategy
}

\author{
Zahid Yaqoob, Jigang Wu, and Changhuei Yang \\ California Institute of Technology, Pasadena CA, USA
}

BioTechniques 39:S6-S13 (December 2005)

doi $10.2144 / 000112090$

\begin{abstract}
This paper reviews the current state of research in spectral domain optical coherence tomography (SDOCT). SDOCT is an interferometric technique that provides depth-resolved tissue structure information encoded in the magnitude and delay of the back-scattered light by spectral analysis of the interference fringe pattern. There are two approaches to SDOCT-one that uses a broadband source and a spectrometer to measure the interference pattern as a function of wavelength and the other that utilizes a narrowband tunable laser that is swept linearly in $k \sim 1 / \lambda$ space during spectral fringe data acquisition. Unlike time domain (TD) OCT, the reference arm is stationary in both SDOCT methods, which allows for ultra high-speed OCT imaging. Owing to its high speed and superior sensitivity, SDOCT has become indispensable in biomedical imaging applications. After a brief introduction and a discussion on sensitivity advantage, methods of implementation of the two SDOCT schemes will be presented. The two peer approaches are compared in speed, scan depth range, complexity, spectral regions of operation, and methods of detection. The review also discusses OCT enhancements and functional methods based on SDOCT format and concludes with possible directions that this research may take in the near future.
\end{abstract}

\section{INTRODUCTION}

Optical coherence tomography (OCT) was first developed by Fujimoto's group at MIT about 10 years ago (1), and the method has since matured into an important clinical imaging modality. The success of OCT in making such a rapid transition from research and development into the clinical setting is not surprising given the numerous advantages that it offers clinicians. (i) Quality images-OCT has demonstrated the ability to render images with $0.5 \mu \mathrm{m}$ resolution (2). (ii) Flexibility - the light fluence level required for OCT imaging is low enough that OCT can be used in sensitive tissue locales, such as the eye (3). Further, OCT probes are typically optical fiber-based, and they may be made to be sufficiently small and pliable to operate within the gastrointestinal (GI) tract (4) and major blood vessels (5). (iii) Additional functionality-while a basic OCT imaging method is able to render depth-resolved structural images of the target, more sophisticated OCT imaging strategies can provide additional functional information, such as flow (through Doppler OCT) (6,7), tissue structural arrangement (through birefringence OCT) $(8,9)$, and the spatial distribution of specific contrast agents (through molecular contrast OCT) $(10,11)$.

Since the invention of OCT, the method has been extensively applied in ophthalmology settings. The popularity of the method lies in its ability to perform high-resolution cross-sectional imaging and analysis of structural changes in the eye during disease progression. For example, OCT is excellent at rendering a depthresolved profile of macular hole disease progression (12), which generally involves a thinning or detachment of the retina and is difficult to assess with en face imaging methods $(13,14)$. OCT has also been proven useful for ophthalmology applications (15-17) where structural information provides an indirect measure of physiologically relevant information that is useful for disease diagnosis. OCT's applications to image other parts of the human body include endoscopic $\mathrm{Gl}$ tract imaging for tumor detection $(4,18)$, monitoring and risk assessing of vulnerable lipid plaques in the vascular system $(19,20)$, monitoring intracoronary stenting $(21)$, and tracking structural weakness associated with tooth decay $(22,23)$. OCT's applications in bioscience endeavors include tracking cardiac development in chick embryos (24), studying gastrulation in mouse embryos (25), and imaging neural morphology in Xenopus laevis (26).

During the past couple of years, there is a general shift in the method by which the research community implements OCT imaging. This shift is brought about by the independent discoveries by a few research groups of the vast increase in sensitivity that an alternate implementation of OCT, spectral domain OCT (SDOCT), has over

6 Molecular Imaging the conventional approach, which is time domain OCT (TDOCT). This alternate method is not new itself; in fact, SDOCT was invented at about the same time as TDOCT (27). However, the method remained largely underutilized and unpopular until the publication of an experimental paper, which experimentally demonstrated that SDOCT has a clear sensitivity advantage over TDOCT (28). This increased sensitivity provided by SDOCT can be parlayed into a higher OCT scan acquisition rate, greater depth penetration, or to boost the sensitivity of the various functional OCT methods.

In the next section, we shall briefly discuss the principles of TDOCT and SDOCT, define a metric for sensitivity determination, and provide a theoretical approach to understand the sensitivity difference. In the third section, we shall present the two approaches for implementing SDOCT. In the fourth section, we will take a brief look at the various research results that make use of the sensitivity advantage to improve specific aspects of the imaging process. In the fifth section, we will discuss the associated issues that have arisen with SDOCT implementations and the methods that various research groups have taken to correct or ameliorate these shortcomings. Finally, we shall conclude with possible directions that SDOCT research may take in the future.

\section{TDOCT AND SDOCT EXPLAINED}

The best way to begin understanding the operating principle of OCT is to compare OCT to ultrasound tomography imaging. Consider the situation where we direct a narrow beam of pulsed light towards a tissue sample. Much like in the case of ultrasound tomography, we can expect to see a train of echoes backscattered from the tissue. By time gating the echo train appropriately, we may then be able to create a depth-resolved line profile of the tissue (or A-scan). By repeating the process at incremental steps across the tissue sample, a two-dimensional (2-D) depth-resolved image (or B-scan) of the sample can be obtained. Unfortunately, the speed of light is too high for existing detectors to be able to respond quickly enough for direct detection of the optical echo pulse train.

Fortunately, all is not lost. The echo pulse train may be indirectly measured by using interferometry. Even better, the use of interferometry frees us from having to use pulsed light sources; as long as the spectral bandwidth of the light source is equal to that of the pulsed light source, the acquired image will be equivalent. While the mental picture of the OCT light source as a pulse light source is unnecessary, it can, nevertheless, be used to provide vital intuitions for understanding OCT. 
We shall now take a closer look at a typical TDOCT system (see Figure 1). For simplicity, we shall assume the light source has a top hat spectral profile ( $k_{o}$ is the mean wavenumber, and $\Delta k$ is the spectral bandwidth in wavenumber). In such a system, the input light source is split into two at the beamsplitter. One component is directed at the sample, and the other is sent to a reference mirror. The backscattered and backreflected signals are then re-channeled and combined through the beam splitter and detected by a photodetector. For any given backscattering interface, we can then derive an expression for the detected power, $P_{\text {detector }}(k)$, at any given wavenumber, $k$, as:

$$
P_{\text {detector }}(k)=P_{r e f}(k)+P_{s i g}(k)+2 \sqrt{P_{s i g}(k) P_{r e f}(k)} \cos \left(k 2\left(x_{r}-x_{s}\right)\right),
$$

where $P_{r e f}(k)$ and $P_{\text {sig }}(k)$ are the collected reference and sample power at wavelength $k$, respectively. $\left(x_{r}-x_{s}\right)$ is the difference in reference arm length and the distance to the sample backscattering interface of interest from the beam splitter.

The total power detected can be derived by integrating over the entire light source spectrum:

$$
P_{\text {detector }}=P_{\text {ref }}+P_{\text {sig }}+2 \sqrt{P_{\text {sig }} P_{\text {ref }}} \sin c\left(\frac{\pi\left(x_{r}-x_{s}\right)}{l_{c}}\right) \cos \left(k_{o} 2\left(x_{r}-x_{s}\right)\right),
$$

where $l_{c}=\frac{\pi}{\Delta k}$ is the coherence length. Note that the interference term (the last term) peaks when the reference arm length is matched to the backscattering interface's distance. The broadness of the peak interference signal is given by the coherence length; to obtain a well-defined determination of the backscattering interface's position, we desire a short coherence length or broad spectral bandwidth from the light source. By detecting the various interference envelop peaks during the scan, the location and backscattering strength of interfaces within the sample can be determined.

From a pulsed light source picture, all of above conclusions are physically intuitive. Interference can only occur when the two arms are matched in length so that the returning pulses can arrive at the detector at the same time to interfere. The sharper the pulse and, hence, the broader the spectral bandwidth, the better we can locate the backscattering interface in the presence of adjacent scattering interfaces.

In a TDOCT system, the reference arm is typically displaced and scanned over a distance equal to the depth range, $x_{\text {depth }}$, that we wish to probe for the sample. For a given total scan time duration, $T$, we can determine that a TDOCT system will spend a duration of $l_{c} x_{c} T$ collecting interference signal from any given interface within the sample.

A determination of the TDOCT sensitivity can be made by calculating the number of useful signal photons within that duration and compare that with the noise photon count. The total useful interference signal photons that will be collected in this context are given by:

$$
\text { Signal }_{\text {TDOCT }}=2 \sqrt{P_{\text {sig }} P_{\text {ref }}} \frac{l_{c} T}{x_{\text {depth }}} \frac{\varepsilon}{h v},
$$

where $\varepsilon$ is the quantum efficiency of the detector and $h v$ is the photon quantum energy. The noise count is given by the square root of the total photons that are detected by the detector during that time duration. As the reference power $P_{\text {ref }}$ typically dominates over all other signal in TDOCT, we get:

$$
\text { Noise }_{T D O C T}=\sqrt{P_{\text {ref }} \frac{l_{c} T}{x_{\text {depth }}} \frac{\varepsilon}{h v}}
$$

The conventional sensitivity figure of merit employed for gauging OCT performance is the signal-to-noise ratio (SNR) quantity given by:

$$
\begin{aligned}
S N R_{\text {TDOCT }} & =10 \log \left(\frac{\text { Signal }_{\text {TDOCT }}}{\text { Noise }_{\text {TDOCT }}}\right)^{2}(\text { unit }: d B) \\
& =10 \log \left(4 P_{\text {sig }} \frac{l_{c} T}{x_{\text {depth }}} \frac{\varepsilon}{h v}\right)
\end{aligned} .
$$

This figure of merit is similar to the shot noise limited sensitivity factor that can be derived for the direct detection of photons with an ideal detector that is free of detector-noise sources. In the context of OCT, it has an additional interpretation. For example, an OCT system that has a SNR of $110 \mathrm{~dB}$ implies that it is sensitive to a reflecting interface in the sample that returns equal or more than 1 part in $10^{11}$ of the incident light. The typical SNR of an OCT system can vary depending on the scan parameters of interest, but a SNR of 80 or above is generally accepted as a minimum required for imaging biological targets.

The form of this figure of merit is worth a closer examination. Though the detector is collecting backscattered photons from the interface of interest for the entire duration of the acquisition, $T$, the detection scheme is only actively selecting those photons for OCT signal construction over a much shorter duration, $\frac{l_{c} T}{x}$. This necessarily leads to diminished signal sensitivity in comparison to a scheme that is capable of using those photons for OCT signal construction over the entire duration of the acquisition. As it turns out, SDOCT is able to make full use of those photons.

A typical spectrometer-based SDOCT scheme is very similar to that of a typical TDOCT scheme. The moving reference mirror is immobilized, and the detector is replaced by a low-loss spectrometer in the SDOCT scheme. In this scheme, the spectral variation of the detected signal as given by Equation 1 is recorded by the spectrometer. Notice that the period of the spectral oscillation in
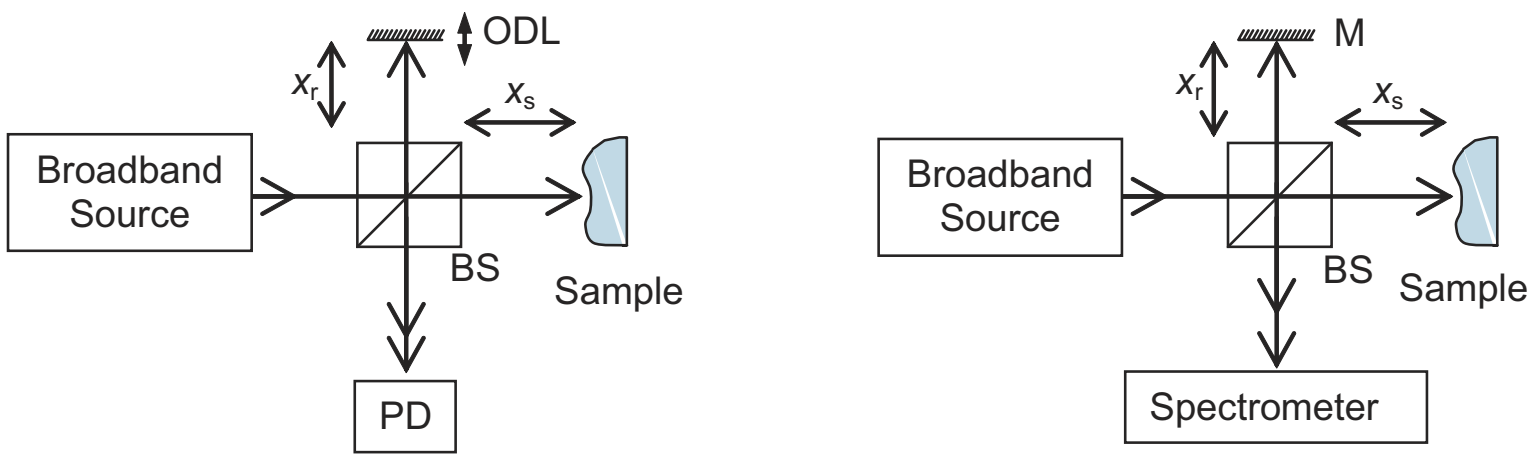

A

Figure 1. Representations of (A) time domain and (B) spectrometer-based spectral domain optical coherence tomography (SDOCT) systems. M, mirror; ODL, optical delay line; PD, photodetector; BS, beamsplitter. 
$k$-space of the measured signal is proportional to $\left(x_{r}-x_{s}\right)$. An interface which gives a smaller $\left(x_{r}-x_{s}\right)$ value will produce a slower sinusoidal spectral oscillation than an interface with a larger $\left(x_{r}-x_{s}\right)$ value. Therefore, a Fourier transform of the spectral measurement will produce a line scan profile similar to that obtained from TDOCT. Notice that in this case, signal contribution from two or more interfaces may be collected simultaneously as they contribute to different spectral oscillation components. In this case, the total useful interference signal photons from a given interface that will be collected in this context are given by:

$$
\text { Signal }_{S D O C T}=2 \sqrt{P_{\text {sig }} P_{\text {ref }}} T \frac{\varepsilon}{h v} \text {, }
$$

[Eq. 6]

where $T$ is the total signal collection time. The noise count is given by the square root of the total photons that are detected by the entire spectrometer during the entire signal collection duration. Therefore we get:

$$
\text { Noise }_{S D O C T}=\sqrt{P_{r e f} T \frac{\varepsilon}{h v}} .
$$

Subsequently, we obtain a SNR measure of:

$$
\begin{aligned}
S_{N D R_{S D C T}} & =10 \log \left(\frac{\text { Signal }_{\text {SDOCT }}}{\text { Noise }_{\text {SDOCT }}}\right)^{2}(\text { unit }: d B) \\
& =10 \log \left(4 P_{\text {sig }} T \frac{\varepsilon}{h v}\right)
\end{aligned}
$$

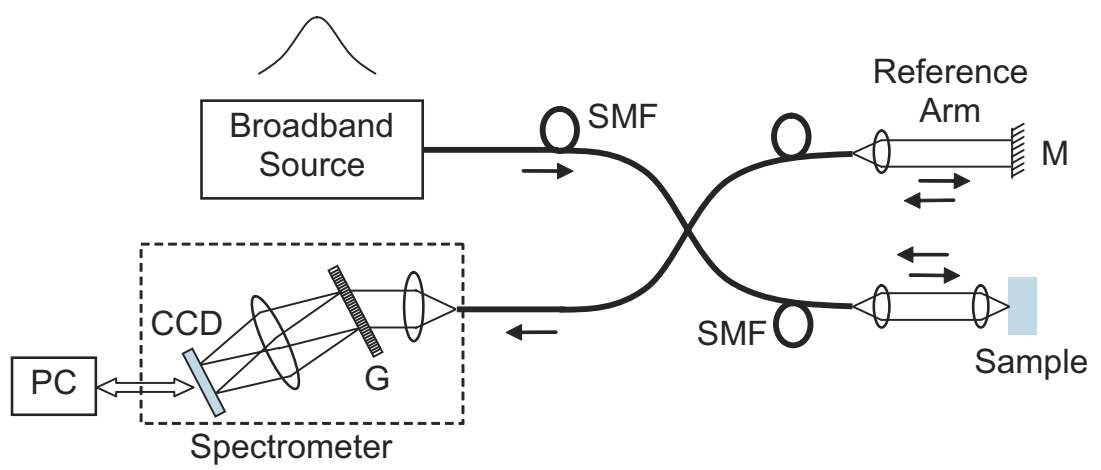

A

B
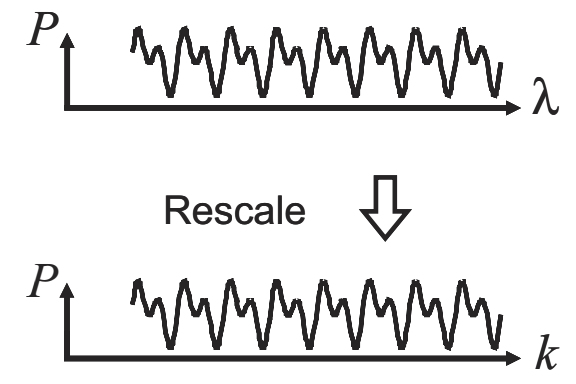

C

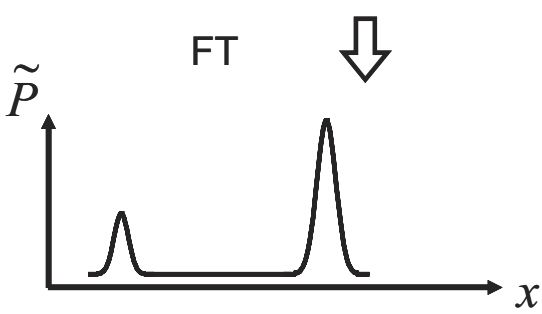

Figure 2. Spectrometer-based spectral domain optical coherence tomography (SDOCT) system where the broadband laser source can be a superluminescent diode (SLD) or a mode-locked ultra-fast laser source. SMF, single mode fiber; G, diffraction grating; PC, personal computer; CCD, charged-coupled device.
The scan depth, $x_{\text {depth }}$, that is achievable with SDOCT is given by the range of spectral oscillation frequency that is detectable by the spectrometer. For a spectrometer with $\mathrm{N}$ pixel, the highest spectral oscillation periodicity that is detectable is $N / 2$. Beyond that point, the oscillation will wrap over and create aliasing signal. Using this criterion, we see that:

$$
\Delta k 2\left(x_{r}-x_{s}\right)_{\max }=\left(\frac{N}{2}\right) 2 \pi \Rightarrow x_{\text {depth }}=\frac{N \pi}{2 \Delta k}=\left(\frac{N}{2}\right) l_{c} .
$$

A comparison of SDOCT and TDOCT is now possible. For a given N pixel-based SDOCT with a depth scan range of $x_{\text {depth }}=\left(\frac{N}{2}\right) l_{c}$, a TDOCT acquiring signal over the same total time duration will have a SNR of:

$$
S N R_{\text {TDOCT }}=10 \log \left(4 P_{\text {sig }}\left(\frac{2}{N}\right) T \frac{\varepsilon}{h v}\right)=S N R_{S D O C T}-10 \log \left(\frac{N}{2}\right) . \quad \text { [Eq. 10] }
$$

Therefore, a SDOCT system is intrinsically more sensitive than a TDOCT system by a factor of N/2. This improvement is attributable to the fact that SDOCT is capable of collecting signals from all depth of the sample during the entire acquisition time. Interested readers may refer to References 29, 30, and 31 for more details on this sensitivity advantage.

\section{METHODS OF IMPLEMENTATION}

Two distinct methods have been developed to implement SDOCT. The first approach, a spectrometer-based SDOCT, also known as spectral radar (32) and Fourier domain OCT $(29,30,33)$, uses a broadband light source and a low-loss spectrometer to measure the spectral oscillations. The second method, sweptsource SDOCT, that has also been termed as optical frequency domain reflectometry (OFDR) (34), wavelength tuning interferometry (WTI) (35), and optical frequency domain imaging (OFDI) (36), employs a rapidly tuned narrowband source to measure spectral oscillations at evenly spaced wavenumbers. In this section, we will discuss the experimental setup of these two methods.

\section{Spectrometer-Based SDOCT System}

The experimental setup of spectrometerbased SDOCT system is shown in Figure $2 \mathrm{~A}$. The setup uses a broadband light source, usually a superluminescent diode (SLD) or mode-locked laser. A $2 \times 2$ fiber-optic coupler forms the Michelson interferometer. In the detector arm, a low-loss spectrometer measures the spectrum of the interference pattern of light returned from the reference and sample arms of the interferometer. The grating-based spectrometer measures the interference signal as a function of wavelength, $\lambda$. Usually, the spectral data is rescaled and resampled evenly in $k$ space, before it is Fourier transformed to get the sample depth profile or A-scan (see Figure 2, $B-D)$. High spatial resolution-a desirable feature of an OCT system-requires the use of a light source with wider spectral width (or very short coherence length $I_{c}$ ). This fact in conjunction with Equation 9 implies that larger depth scan range would require a higher number of spectrometer's linear array (CCD camera) pixels, $N$. This is also supported by the argument that by decreasing the pixel size and increasing the number of CCD 
pixels $N$, one can increase the highest detectable spectral oscillation which will correspond to a larger scan range.

\section{Swept Source SDOCT System}

In swept source SDOCT system, shown in Figure 3, the source is a rapidly tunable narrowband laser. The output of the source can be written as $S[k(t)]$. Usually $k(t)$ is swept linearly and can be written as $k(t)=\mathrm{k}_{0}+\delta k \times \mathrm{t}$, where $\delta \mathrm{k}=\Delta k / \Delta t$ such that $\Delta k$ is the total optical bandwidth through which the narrowband source is swept, and $\Delta t$ is the total sweep time. A swept source SDOCT system ideally acquires signal at $M$ evenly spaced wavenumbers, such that $M \times \delta k=\Delta k$. In this context, a swept source SDOCT system will have a depth scan range $x_{\text {depth }}=\frac{M \pi}{2 \Delta k}=\frac{\pi}{2 \delta k}=\frac{M}{2} l_{c}$. The interference signal $\mathrm{D}\left[k_{m}\right]$ can be detected by a single detector or dual balanced detectors (as shown in Figure 3 ) to compensate for intensity fluctuations. As the signal is acquired at evenly spaced wavenumbers, $\mathrm{D}\left[k_{m}\right]$ can be discrete Fourier transformed (DFT) directly to derive the depth-resolved OCT line scan of the tissue sample under study.

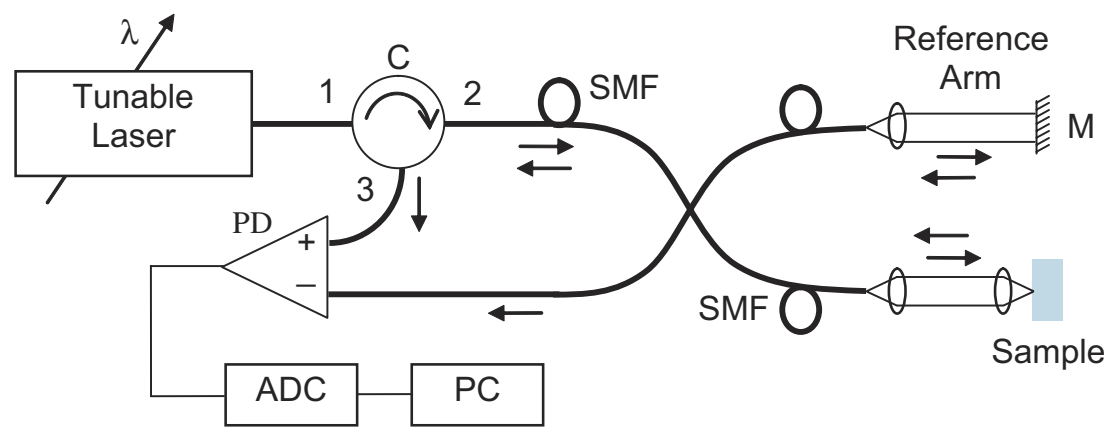

Figure 3. Swept source-based spectral domain optical coherence tomography (SDOCT) system. SMF, single mode fiber; C, optical circulator; M, mirror; PD, photodetector; ADC, analog-to-digital converter; PC, personal computer.
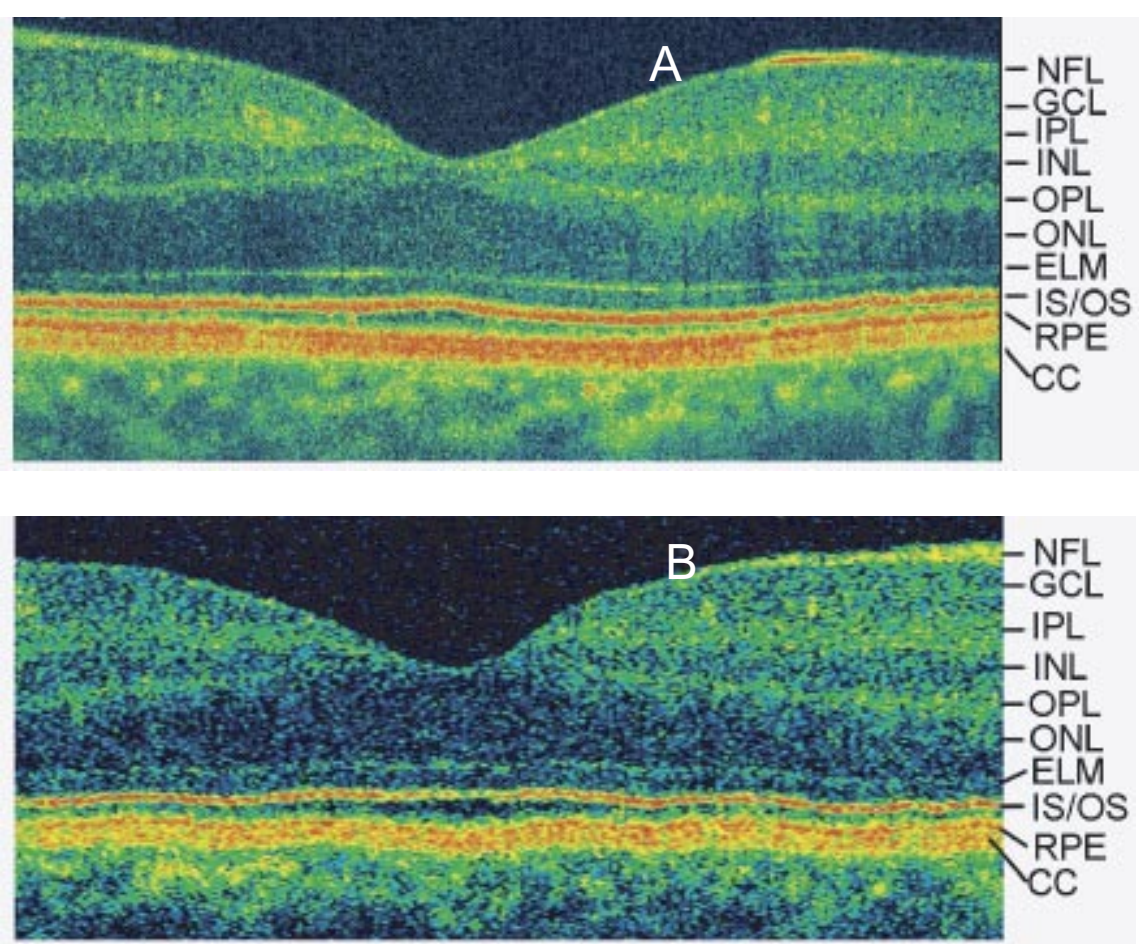

Figure 4. Human retinal images using (A) a spectrometer-based spectral domain optical coherence tomography (SDOCT) system and (B) time domain optical coherence tomography (TDOCT) system. NFL, nerve fiber layer; GCL, ganglion cell layer; IPL, inner plexiform layer; INL, inner nuclear layer; OPL, outer plexiform layer; ONL, outer nuclear layer; ELM, external limiting membrane; IS/OS, photoreceptor inner and outer segment junction; RPE, retinal pigment epithelium; CC, choriocapillaris. Reprinted with permission from Reference 38 .

\section{ADVANTAGES OF SDOCT}

biomedical community has seen a continuous string of sources. The increased bandwidth in turn leads to decreased coherence length and enables the acquisition of ultra high axia OCT images $(2,37)$. In a TDOCT system, increasing electronic detection bandwidth. In order to maintain the same SNR, one has to either decrease the A-scan rate or depth scan range, or increase the sample illumination intensity. As a matter of fact, both as in ophthalmic imaging, the incident optical power cannot be increased beyond the American National Standards Institute (ANSI) SDOCT systems show significant sensitivity advantage over the TDOCT. Therefore, SDOCT systems may be used to perform OCT imaging at higher speed and (or) scan depth.

Several research groups have demonstrated high-resolution SDOCT imaging at remarkably high speeds. Using a $144 \mathrm{~nm}$ fullwidth-half-maximum (FWHM) spectral width broadband light source centered at $825 \mathrm{~nm}$, M. Wojtkowski et al. (38) demonstrated a highresolution (2.1 $\mu \mathrm{m}$ axial resolution in tissue) spectrometer-based SDOCT system with 98 $\mathrm{dB}$ SNR for $750 \mu \mathrm{W}$ incident optical power and high (16,000 A-scans rate at 1024 pixels per A-scan) imaging speed. Figure 4A shows a high-resolution retinal image comprising of 3000 A-scans acquired in $150 \mathrm{~ms}$ with a high-speed SDOCT system. For comparison purposes, a high axial resolution retinal image comprising of $300 \mathrm{~A}$-scans acquired in $2 \mathrm{~s}$ by a TDOCT is shown in Figure 4B. Thus, an increase in the imaging speed in SDOCT systems allows acquiring higher number of depth scans to deliver superior resolution in the transverse direction-a desired feature for optimum resolution not possible with TDOCT systems. In another work, video-rate spectrometer-based SDOCT imaging at 29 frames/s with 1000 A-scans/frame and 1024 pixels/A-scan reported by B. Cense et al. (39) is regarded as the best OCT imaging performance (in terms of speed) accounted so far.

As mentioned earlier, swept source SDOCT acquires interferometric data from a sample as the wavelength of tunable laser is adjusted linearly in time. As wider optical wavelength sweep range will deliver higher depth resolution and faster wavelength tuning of the source will permit rapid data acquisition, the design and functionality of tunable source plays important role in the performance of swept source-based SDOCT system. In recent years, various laser designs in 1300 $\mathrm{nm}$ band have been demonstrated for swept source SDOCT. Demonstrated tunable light sources are based on galvanometer scanning mirror $(36,40)$, polygon rotating mirror (41), and tunable Fabry-Perot (FP) cavity (30). Using a single polygon mirror scanner, Oh et al. reported a dual laser cavity swept source 
Table 1. Comparison Between Swept Source and Spectrometer-Based SDOCT

\begin{tabular}{|lll|}
\hline & Swept Source SDOCT & Spectrometer-Based SDOCT \\
\hline SNR drop-off & No & Yes \\
Spectral band (nm) & 1300 & 800 \\
Heterodyne detection & Yes & No \\
Balanced detection & Yes & No \\
Speed & Comparable & \\
MCOCT & No & Yes \\
OCT system & Simple & Complex \\
Light source & Complex & Simple \\
SNR, signal-to-noise; SDOCT, spectral domain optical coherence tomography; MCOCT, molecular \\
contrast optical coherence tomography.
\end{tabular}

two arms of the interferometer or the fixed material in front of the imaging range. In case of retinal imaging, a major part of dispersion arises from the vitreous that forms the majority of the eye length. Nevertheless, the two arms of the interferometer should be balanced for the dispersive media to achieve optimum depth resolution.

In conventional OCT, dispersion artifacts can be compensated for by introducing glass such as BK7 or water in the reference arm. In the case of SDOCT, another approach exists for dispersion compensation-through software. Recently, two independent research groups at Harvard Medical School (Boston, MA) and Massachusetts Institute of Technology

with $145 \mathrm{~nm}$ tuning range around $1310 \mathrm{~nm}$ and at $20 \mathrm{kHz}$ repetition rate (42). The peak and average optical power were limited to 1.8 and $1.35 \mathrm{~mW}$, respectively. The output optical power of these systems were relatively low. Recently, R. Huber et al. demonstrated a swept source with $120 \mathrm{~nm}$ tunable bandwidth centered at $1310 \mathrm{~nm}$ (43) based on the use of a tunable FP cavity. This swept source is capable of delivering approximately $45 \mathrm{~mW}$ output optical power at $20 \mathrm{kHz}$ sweep rate. Figure 5, A and B, show swept source SDOCT images at 20 and $27 \mathrm{kHz}$, respectively. In conclusion, swept sourceand spectrometer-based SDOCT systems are comparable in imaging speed.

In addition to comparable imaging performance, swept source systems feature reduced excess noise. This is because at each wavenumber $k$, the output of the swept source is a narrowband laser with much smaller noise than that of the broadband source. Further, due to the nature of detection in swept source SDOCT, we do not see the SNR drop-off that usually occurs in spectrometerbased systems. Swept source SDOCT also allows heterodyne detection-an added feature that shifts the interferometric signal frequency spectrum away from the zero frequency permitting detection of positive as well as negative displacements (44).

Dispersion is a phenomenon that degrades the quality of OCT images and is severe when ultra broadband sources are employed. Due to the wavelength dependence of propagation constant $k(\lambda)=k_{0} n(\lambda)$, certain spectral components of the broadband light slow down relative to one another as the light passes through a medium. Since different optical path lengths corresponding to different spectral components within the medium can be compensated for at different positions of the reference mirror, the effective width of the coherence function is increased-thus, compromising the axial resolution. Usually, the dispersion over the axial depth range is negligible and is mainly due to unbalanced optics in the
(MIT; Cambridge, MA) have discussed numerical methods for dispersion compensation $(38,39)$. Figure 6 shows retinal images with and without dispersion compensation. Figure $6, \mathrm{~B}$ and $\mathrm{C}$, show a comparison of dispersion compensation through hardware and software, respectively.

Motions in the sample or probe can cause signal fading, decrease in axial and (or) transversal resolution, and reduction in SNR, which are manifested as image distortion and blurring. In TDOCT, probeor sample-related motion affects only the image pixel for which the signal is being acquired. If the signal acquisition time per pixel is very small, the degradation in SNR and spatial resolution due to motion may be negligible. However, motion-induced image degradation in SDOCT systems, in which signal is acquired over time from various depths of interest inside the sample, may be significant. The problem can be alleviated by increasing the OCT imaging speed-a focus of recently reported work. In spectrometer-based SDOCT systems, the motional artifacts can be further reduced by decreasing the CCD illumination time. This can be achieved by using a pulsed instead of a $c w$ broadband source (45).

Spectroscopic OCT is an enhancement of standard OCT and provides spatially resolved spectroscopic information of the sample (46). This added information about depth resolved absorption can lead to enhanced image contrast allowing differentiation of tissue pathologies. In TDOCT, the interferometric data corresponding to each pixel is processed to retrieve the spectroscopic information. In the case of SDOCT, there is a direct access to the spectroscopic information (47). This shows that SDOCT systems can lead to a simple and fast way to investigate depth resolved spectral signatures of a biological sample.

Molecular contrast OCT (MCOCT) is a functional OCT technique that combines the major advantages of both fluorescence microscopy (chemical contrast detection and imaging capability)

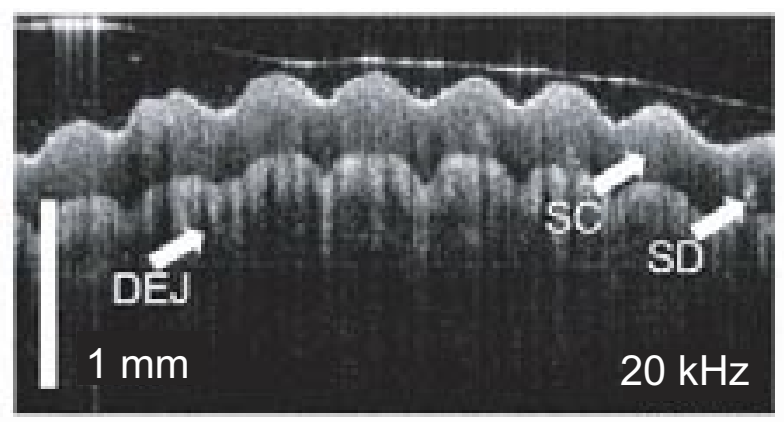

A

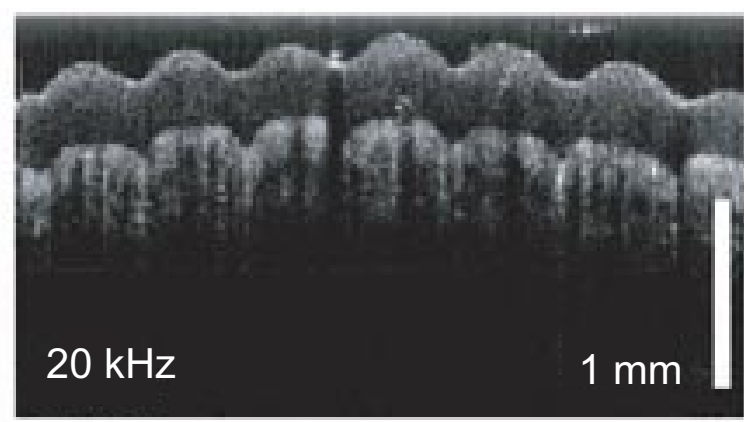

B

Figure 5. Human skin in the area of finger tip imaged at (A) $20 \mathrm{kHz}$ and (B) $27 \mathbf{k H z}$. DEJ, dermal epidermal junction; SC, stratum corneum; SD, sweat ducts. Reprinted with permission from Reference 43. 

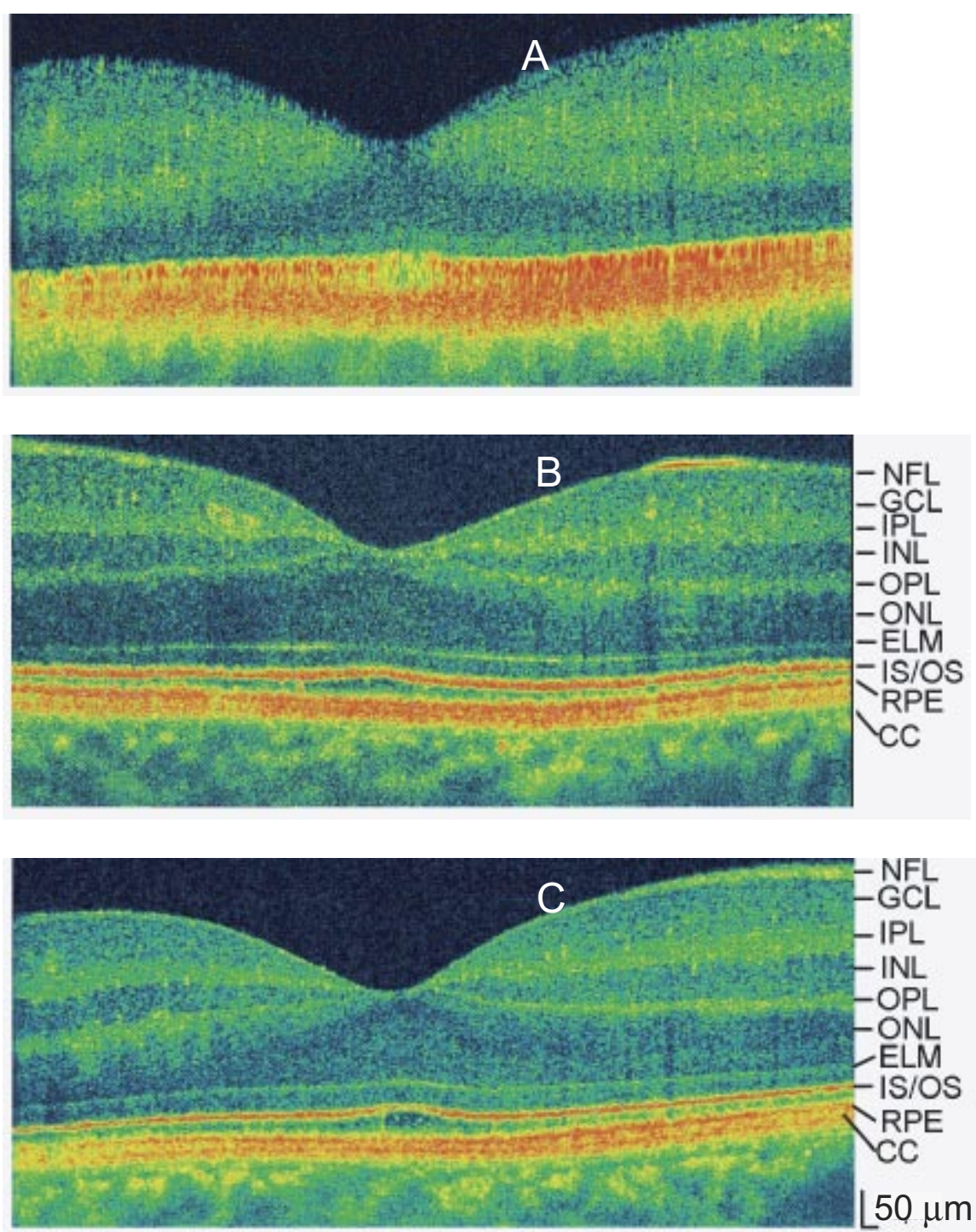

Figure 6. Retinal images with and without dispersion compensation. (A) Retinal image without dispersion compensation. (B and C) Retinal images with dispersion compensation using a water cell in the reference arm and through software, respectively. NFL, nerve fiber layer; GCL, ganglion cell layer; IPL, inner plexiform layer; INL, inner nuclear layer; OPL, outer plexiform layer; ONL, outer nuclear layer; ELM, external limiting membrane; IS/OS, photoreceptor inner and outer segment junction; RPE, retinal pigment epithelium; CC, choriocapillaris. Reprinted with permission from Reference 38.

\section{Gill pockets}

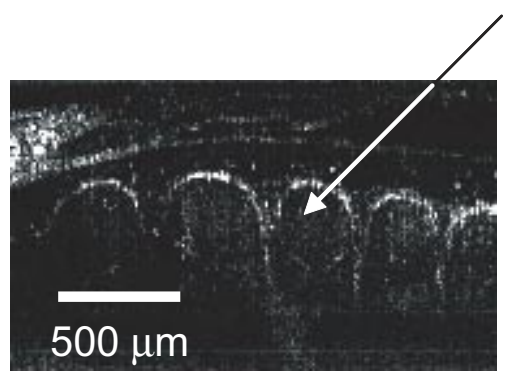

A

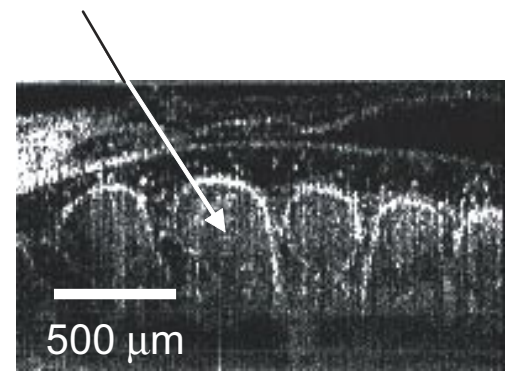

$B$
Figure 7. Molecular contrast optical coherence tomography (MCOCT) imaging of a stage 54 Xenopus laevis gill pockets filled with a mixture of $400 \mu \mathrm{M}$ ICG, $0.125 \% 0.1 \mu \mathrm{m}$ latex microspheres, and $\mathbf{0 . 6 \%}$ agarose. (A and B) show OCT images before and after the ICG photobleaching, respectively. ICG, indocyanine green. and OCT (higher spatial resolution and depth range) (11). Although, most MCOCT methods reported so far have been implemented using TDOCT, they can be adapted to SDOCT format for higher sensitivity advantage. Recently, a pump-probe scheme for MCOCT was implemented using spectrometer-based SDOCT (48). The scheme used indocyanine green (ICG), an FDA-approved dye with an absorption spectrum that peaks at $790 \mathrm{~nm}$, as a contrast agent. An OCT scan is first performed followed by photobleaching of the ICG. Then a second OCT scan is acquired. The difference of the two OCT scans yields information about the depth resolved distribution of the contrast agent (ICG) within the sample. Mapping of ICG distribution within the gill pockets of a stage 54 Xenopus laevis using the above spectral domain MCOCT technique is shown in Figure 7.

\section{ISSUES ASSOCIATED WITH SDOCT}

Despite many advantages discussed in the previous section, there are some issues that have arisen with the implementation of SDOCT. In this section, we will briefly discuss these issues and steps that different research groups have taken to overcome them.

\section{SNR Drop-Off}

The SNR drop-off in SDOCT systems is caused by the finite wavelength resolution during the signal acquisition. In the swept source SDOCT systems, the linewidth corresponding to each wavenumber is so small that this factor can be neglected. The SNR drop-off in swept source SDOCT systems is mainly caused by the nonlinearities in the wavenumber scan (30). Usually the nonlinearities in the swept source are not conspicuous, so the SNR drop-off is not prominent and therefore can be ignored over the depth scan range.

In spectrometer-based SDOCT systems, the SNR is not the same through the whole depth scan range. Instead, it drops as the pathlength difference between reference and sample arms increases. The SNR dropoff can be described as the convolution of the spectral resolution function, which is a rect function of the finite pixel width of the $C C D$, with the interference signal in the frequency domain (29). In the spectrometerbased SDOCT system, the SNR drop-off can be as serious as approximately $20 \mathrm{~dB}$ over the scan range. This problem can be ameliorated by either decreasing the pixel size of the CCD camera or moving the interested region of the sample near the equalpathlength point. Recently, Z. Hu et al. (49) reported an improvement in the SNR drop- 


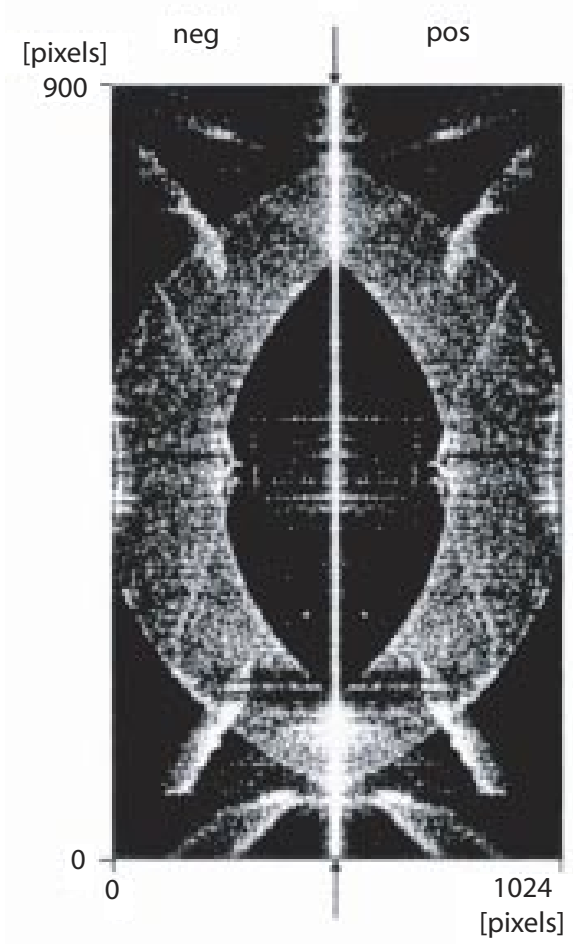

A
Optical Path Difference

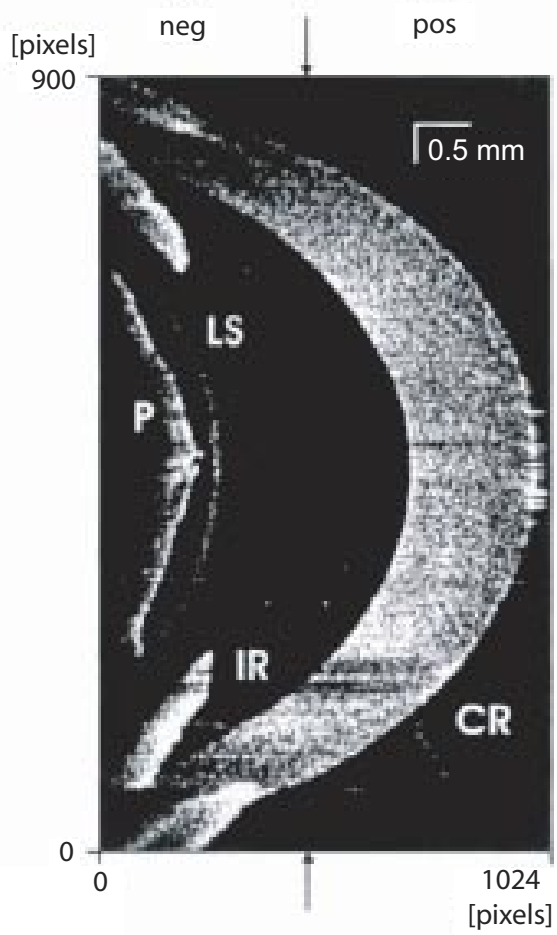

B

Figure 8. Porcine eye images. (A) Porcine eye image using standard spectral domain optical coherence tomography (SDOCT). Mirror image is caused by symmetrical nature of Fourier transform (FT) of real function. (B) Porcine eye image without wrapping due to complex signal acquisition. neg, negative; pos, positive. Reprinted with permission from Reference 51.

off by acquiring two sets of spectra; one before and another after shifting the CCD camera by a distance of half pixel size.

\section{Mirror Image}

Since the spectrum acquired in SDOCT is a real function, its Fourier transform (FT) is symmetrical with respect to the equalpathlength point. The symmetrical nature of FT causes a mirror image that limits the overall scan range in SDOCT. The way to avoid mirror image and hence double the scan range is to get the full-range complex signal (50). To implement this, the phase information of the interference signal must be retrieved. Many methods have been developed to achieve this goal. One approach is the five-frame method, in which the complex signal is constructed by taking five consecutive measurements of the spectra taken with a phase shift in increments of $\pi / 2$ (51). Figure 8 shows porcine eye images using standard and complex SDOCT. It can be seen that the complex signal gives an image without wrapping. Other methods include using a $3 \times 3$ fiber-optic coupler in the interferometer (52), translation of the reference mirror to introduce a $90^{\circ}$ phase shift (53), and the application of an electro-optic phase modulator (54) to acquire the complex OCT spectrum.

\section{FUTURE DIRECTIONS}

As shown in the previous sections, the swept source SDOCT system has many advantages, such as reduced excess noise, no SNR drop-off, and heterodyne detection capability, over spectrometer-based SDOCT system (see Table 1 for comparison). However, all reported implementations of swept sources have been realized in the $1300 \mathrm{~nm}$ band. In some cases, it might be preferable to do the imaging in other wavelength bands. For instance, it was reported that $1040 \mathrm{~nm}$ light is more suitable for retinal imaging because it will enhance penetration into the choroids (55). Similarly, most of the molecular dyes such as ICG have absorption bands in the $800 \mathrm{~nm}$ regime. Therefore, to enjoy the advantages of swept source SDOCT, it would be highly desired to realize highspeed, broadly tunable swept sources in other wavelength bands.

Another worthy research direction is to investigate ways to resolve the problem of SNR drop-off in spectrometer-based SDOCT systems that severely limits the scan depth range to approximately $2 \mathrm{~mm}$ or less. Until now, there is no efficient method reported to significantly improve the SNR drop-off.

Finally, with the greatly increased data input rate that SDOCT provides and the computationally intensive FTs that SDOCT requires, it has become important to introduce high-speed signal processing modules, such as field programmable gate arrays (FPGAs) to acquire and process the OCT signal in real time for true video-rate imaging.

\section{COMPETING INTERESTS STATEMENT}

The authors declare no competing interests.

\section{REFERENCES}

1. Huang, D., E.A. Swanson, C.P. Lin, J.S. Schuman, W.G. Stinson, W. Chang, M.R. Hee, T. Flotte, et al. 1991. Optical coherence tomography. Science 254:1178-1181

2. Povazay, B., K. Bizheva, A.H. Unterhuber, B. Hermann, H. Sattmann, A.F. Fercher, W. Drexler, A. Apolonski, et al. 2002. Submicrometer axial resolution optical coherence tomography. Opt. Lett. 27:1800.

3. Swanson, E.A., J.A. Izatt, M.R. Hee, D. Huang, C.P. Lin, J.S. Schuman, C.A. Puliafito, and J.G. Fujimoto. 1993. In-vivo retinal imaging by optical coherence tomography. Opt. Lett. 18:1864-1866.

4. Rollins, A.M., R. Ung-arunyawee, A. Chak, R.C.K. Wong, K. Kobayashi, M.V. Sivak, and J.A. Izatt. 1999. Real-time in vivo imaging of human gastrointestinal ultrastructure by use of endoscopic optical coherence tomography with a novel efficient interferometer design. Opt. Lett. 24:1358-1360.

5. Chen, Z.P., T.E. Milner, S. Srinivas, X.J. Wang, A. Malekafzali, M.J.C. vanGemert, and J.S. Nelson. 1997. Noninvasive imaging of in vivo blood flow velocity using optical Doppler tomography. Opt. Lett. 22:1119-1121.

6. Izatt, J.A., M.D. Kulkarni, S. Yazdanfar, J.K. Barton, and A.J. Welch. 1997 In vivo bi-directional color Doppler flow imaging of picoliter blood volumes using optical coherence tomography. Opt. Lett. 22:4139-4141.

7. Yazdanfar, S., A.M. Rollins, and J.A. Izatt. 2000. Imaging and velocimetry of the human retinal circulation with color Doppler optical coherence tomography. Opt. Lett. 25:1448-1450.

8. deBoer, J.F., T.E. Milner, M.J.C. vanGemert, and J.S. Nelson. 1997. Twodimensional birefringence imaging in biological tissue by polarization-sensitive optical coherence tomography. Opt. Lett. 22:934-936.

9. Everett, M.J., K. Schoenenberger, B.W. Colston, and L.B. Da Silva. 1998 Birefringence characterization of biological tissue by use of optical coherence tomography. Opt. Lett. 23:228-230

10. Rao, K.D., M.A. Choma, S. Yazdanfar, A.M. Rollins, and J.A. Izatt. 2003 Molecular contrast in optical coherence tomography by use of a pump-probe technique. Opt. Lett. 28:340-342.

11. Yang, C.H. 2005. Molecular contrast optical coherence tomography: a review. Photochem. Photobiol. 81:215-237. 
12. Puliafito, C.A., M.R. Hee, C.P. Lin, E. Reichel, J.S. Schuman, J.S. Duker, J.A. Izatt, E.A. Swanson, and J.G. Fujimoto. 1995. Imaging of macular diseases with optical coherence tomography. ophthalmology 102:217-229.

13. Podoleanu, A.G., G.M. Dobre, and D.A. Jackson. 1998. En-face coherence imaging using galvanometer scanner modulation. Opt. Lett. 23:147-149.

14.Podoleanu, A.G., G.M. Dobre, D.J. Webb, and D.A. Jackson. 1997 Simultaneous en-face imaging of two layers in the human retina by low-coherence reflectometry. Opt. Lett. 22:1039-1041.

15. Targowski, P., M. Wojtkowski, A. Kowalczyk, T. Bajraszewski, M. Szkulmowski, and W. Gorczynska. 2004. Complex spectral OCT in human eye imaging in vivo. Opt. Commun. 229:79-84.

16. Kaluzny, J.J., M. Wojtkowski, and A. Kowalczyk. 2002. Imaging of the anterior segment of the eye by spectral optical coherence tomography. Optica Applicata 32:581-589.

17. Wojtkowski, M., A. Kowalczyk, P. Targowski, and I. Gorczynska. 2002. Frequency domain optical coherence tomography techniques in eye imaging. Acta Physica Polonica A 102:739-746.

18. Sivak, M.V., K. Kobayashi, J.A. Izatt, A.M. Rollins, R. Ung-runyawee, A. Chak, R.C.K. Wong, G.A. Isenberg, and J. Willis. 2000. High-resolution endoscopic imaging of the $\mathrm{Gl}$ tract using optical coherence tomography. Gastrointest. Endosc. 51:474-479.

19. Tearney, G. and B. Bouma. 2002. Atherosclerotic plaque characterization by spatial and temporal speckle pattern analysis. Opt. Lett. 27:533-535

20. Yabushita, H., B.E. Bouma, S.L. Houser, T. Aretz, I. Jang, K.H. Schlendorf, C.R. Kauffman, M. Shishkov, et al. 2003. Monitoring atherosclerotic plaque evolution in vivo by optical coherence tomography. Circulation 108:416.

21. Bouma, B.E., G.J. Tearney, H. Yabushita, M. Shishkov, C.R. Kauffman, D.D. Gauthier, B.D. MacNeill, S.L. Houser, et al. 2003. Evaluation of intracoronary stenting by intravascular optical coherence tomography. Heart 89:317-320

22. Wang, X.J., T.E. Milner, J.F. de Boer, Y. Zhang, D.H. Pashley, and J.S. Nelson. 1999. Characterization of dentin and enamel by use of optical coherence tomography. Appl. Opt. 38:2092-2096.

23. Brandenburg, R., B. Haller, and C. Hauger. 2003. Real-time in vivo imaging of dental tissue by means of optical coherence tomography (OCT). Opt. Commun. 227:203-211

24. Yelbuz, T.M., M.A. Choma, L. Thrane, M.L. Kirby, and J.A. Izatt. 2002. Optical coherence tomography - a new high-resolution imaging technology to study cardiac development in chick embryos. Circulation 106:2771-2774.

25. Perea-Gomez, A., A. Camus, A. Moreau, K. Grieve, G. Moneron, A. Dubois, C. Cibert, and J. Collignon. 2004. Initiation of gastrulation in the mouse embryo is preceded by an apparent shift in the orientation of the anterior-posterior axis. Curr. Biol. 14:197-207.

26. Boppart, S.A., B.E. Bouma, M.E. Brezinski, G.J. Tearney, and J.G. Fujimoto. 1996. Imaging developing neural morphology using optical coherence tomography. J. Neurosci. Methods 70:65-72.

27. Fercher, A.F., C.K. Hitzenberger, G. Kamp, and S.Y. Elzaiat. 1995 Measurement of intraocular distances by backscattering spectral interferometry. Opt. Commun. 117:43-48

28. Wojtkowski, M., R. Leitgeb, A. Kowalczyk, T. Bajraszewski, and A.F. Fercher. 2002. In vivo human retinal imaging by Fourier domain optical coherence tomography. J. Biomed. Opt. 7:457-463.

29. Leitgeb, R., C.K. Hitzenberger, and A.F. Fercher. 2003. Performance of Fourier domain vs. time domain optical coherence tomography. Opt. Express 11:889894.

30. Choma, M.A., M. Sarunic, C. Yang, and J.A. Izatt. 2003. Sensitivity advantage of swept source and Fourier domain optical coherence tomography. Opt. Express 11:2183-2189

31. de Boer, J.F., B. Cense, B.H. Park, M.C. Pierce, G.J. Tearney, and B.E. Bouma. 2003. Improved signal-to-noise ratio in spectral-domain compared with time-domain optical coherence tomography. Opt. Lett. 28:2067-2069.

32. Hausler, G. and M.W. Lindner. 1998. "Coherence radar" and "spectral radar"new tools for dermatological diagnosis. J. Biomed. Opt. 3:21.

33. Wojtkowski, M., A. Kowalczyk, P. Targowski, and I. Gorczynska. 2002. Fourier-domain optical coherence tomography: next step in optical imaging. Optica Applicata 32:569-580.

34. Golubovic, B., B.E. Bouma, G.J. Tearney, and J.G. Fujimoto. 1997. Optical frequency-domain reflectometry using rapid wavelength tuning of a $\mathrm{Cr} 4+$ forsterite laser. Opt. Lett. 22:1704-1706

35. Lexer, F., C.K. Hitzenberger, A.F. Fercher, and M. Kulhavy. 1997. Wavelengthtuning interferometry of intraocular distances. Appl. Opt. 36:6548-6553.

36. Yun, S.H., G.J. Tearney, J.F. de Boer, N. Iftimia, and B.E. Bouma. 2003. High-speed optical frequency-domain imaging. Opt. Express 11:2953-2963.

37. Drexler, W., U. Morgner, F.X. Kartner, C. Pitris, S.A. Boppart, X.D. Li, E.P. Ippen, and J.G. Fujimoto. 1999. In vivo ultrahigh-resolution optical coherence tomography. Opt. Lett. 24:1221-1223.

38. Wojtkowski, M., V.J. Srinivasan, T. Ko, J.G. Fujimoto, A. Kowalczyk, and J.S. Duker. 2004. Ultrahigh-resolution, high-speed, Fourier domain optical coherence tomography and methods for dispersion compensation. Opt. Express 12:2404.
39. Cense, B., N.A. Nassif, T.C. Chen, M.C. Pierce, S.-H. Yun, B.H. Park, B.E. Bouma, G.J. Tearney, and J.F. de Boer. 2004. Ultrahigh-resolution high-speed retinal imaging using spectral-domain optical coherence tomography. Opt. Express 12:2435-2447

40. Yun, S.H., C. Boudoux, M.C. Pierce, J.F. de Boer, G.J. Tearney, and B.E. Bouma. 2004. Extended-cavity semiconductor wavelength-swept laser for biomedical imaging. leee Photonics Technology Letters 16:293-295.

41.Yun, S.H., C. Boudoux, G.J. Tearney, and B.E. Bouma. 2003. High-speed wavelength-swept semiconductor laser with a polygon-scanner-based wavelength filter. Opt. Lett. 28:1981.

42. Oh, W.Y., S.H. Yun, G.J. Tearney, and B.E. Bouma. 2005. Wide tuning range wavelength-swept laser with two semiconductor optical amplifiers. leee Photonics Technology Letters 17:678-680.

43. Huber, R., M. Wojtkowski, K. Taira, J.G. Fujimoto, and K. Hsu. 2005. Amplified frequency swept lasers for frequency domain reflectometry and OCT imaging: design and scaling principles. Opt. Express 13:3513-3528.

44. Yun, S.H., G.J. Tearney, J.F. de Boer, and B.E. Bouma. 2004. Removing the depth-degeneracy in optical frequency domain imaging with frequency shifting. Opt. Express 12:4822-4828

45. Yun, S.H., G.J. Tearney, J.F. de Boer, and B.E. Bouma. 2004. Pulsed-source and swept-source spectral-domain optical coherence tomography with reduced motion artifacts. Opt. Express 12:5614-5624.

46. Morgner, U., W. Drexler, F.X. Kartner, X.D. Li, C. Pitris, E.P. Ippen, and J.G. Fujimoto. 2000. Spectroscopic optical coherence tomography. Opt. Lett. 25:111-113.

47. Leitgeb, R., M. Wojtkowski, A. Kowalczyk, C.K. Hitzenberger, M. Sticker, and A.F. Fercher. 2000. Spectral measurement of absorption by spectroscopic frequency-domain optical coherence tomography. Opt. Lett. 25:820-822.

48. Yaqoob, Z., J. Wu, J.P. Fingler, S. Lintner, B.E. Applegate, and C. Yang. 2005 Pump-probe scheme for optical coherence tomography using indocyanine green mixed with albumin or human plasma. Conference on Lasers and Electro-Optics (CLEO 2005), paper no. CFA3, 22-27 May. Baltimore, MD.

49. Hu, X., A.M. Rollin, and J.A. Izatt. 2005. Enhancement of FDOCT imaging range by sub-pixel spectral shifting. Conference on Lasers and Electro-Optics (CLEO 2005), paper no. CFA7, 22-27 May. Baltimore, MD.

50. Fercher, A.F., R. Leitgeb, C.K. Hitzenberger, H. Sattmann, and M. Wojtkowski. 1999. Complex spectral interferometry OCT. Proc. SPIE 3564:173-178

51. Wojtkowski, M., A. Kowalczyk, R. Leitgeb, and A.F. Fercher. 2002. Full range complex spectral optical coherence tomography technique in eye imaging. Opt. Lett. 27:1415-1417.

52. Choma, M.A. 2003. Instantaneous quadrature low-coherence interferometry with $3 \times 3$ fiber-optic couplers. Opt. Lett. 28:2162-2164.

53. Leitgeb, R.A., C.K. Hitzenberger, A.F. Fercher, and T. Bajraszewski. 2003 Phase-shifting algorithm to achieve high-speed long-depth-range probing by frequency-domain optical coherence tomography. Opt. Lett. 28:2201-2203.

54.Zhang, J., J.S. Nelson, and Z.P. Chen. 2005. Removal of a mirror image and enhancement of the signal-to-noise ratio in Fourier-domain optical coherence tomography using an electro-optic phase modulator. Opt. Lett. 30:147-149.

55. Unterhuber, A., B. Povazay, B. Hermann, H. Sattmann, A. Chavez-Pirson and W. Drexler. 2005. In vivo retinal optical coherence tomography at 1040 nm-enhanced penetration into the choroid. Opt. Express 13:3252-3258.

Address correspondence to:

Changhuei Yang

Electrical Engineering Department and Bioengineering Department

Engineering and Applied Sciences Division

California Institute of Technology,

Pasadena, CA 91125, USA

e-mail: chyang@caltech.edu 